\title{
Assessing Environmental Orientations of Children at UNRWA Schools: Investigating Psychometric Properties of the New Ecological Paradigm Scale
}

\author{
Imad Tawfiq Sa'di \\ Associate Professor at Faculty of Education \\ Yarmouk University, Irbid, Jordan
}

\begin{abstract}
The present study developed a modified New Ecological Paradigm (NEP) Scale for evaluating the multidimensional nature of environmental orientations following a descriptive correlational research design. The participants of the study were 325 second and third grade students of both genders at UNRWA schools, Jordan. They responded to the NEP to investigate their perceptions about ecology, environment and the causes affecting their quality, which is of interest to environmental education curriculum designers for school children. The results have shown a significant and positive impact of the rights of nature, eco-crisis and Human Exemptionalism. The NEP scale has been regarded as a valuable tool for increasing the response rate, reducing the response burden, and improving the completion rate to check the validity, reliability, and dimensionality of the study tool. Therefore, the scale was recommended to be used as a valid instrument to assess lower elementary grade students' environmental orientations and thereby evaluating the effectiveness of the environmental curriculum in developing such orientations.
\end{abstract}

Keywords: Jordan; elementary school children; new ecological paradigm; psychometric properties; UNRWA schools.

\section{Introduction}

The environmental issues are rapidly paving in underdeveloped, developing, and developed countries as the outcomes of environmental damages have become so horrifying and apparent. Studies show that there are several modes to the human behaviors that improve or worsen the quality of the natural environment (Al Menhali, Khalid \& Iyanna, 2018; Barakat, 2015). They depend on subjective measures of attitudes, emotions and perception with comparison to valid, reliable and objective measures. For instance, from pollution to global climate change, from environmental issues to threatening of the individuals, 
depletion of communities, living organisms on the planet, and the natural resources. These all are the most crucial social problems of the present ecological society (Danczyk, Jacobs, Montgomery, Jenkins, \& Farry, 2017).

Individuals will be competent to overcome the environmental crisis that defies a balanced environment through an environmental conscious society. This statement has its consequences on the assumption of many environmentalists that individuals who are more environmentally aware and knowledgeable may teach, motivate and acquaint others how to deal with the environment and its problems (Halkos, Gkargkavouzi, \& Matsiori, 2018). Additionally, environmental orientations play an important contribution in the environmental behavior of individuals. Thereby, the core objective of environmental orientations and the programs associated with them is to pave the environmental knowledge and consequently environmentally friendly behavior (Miller et al., 2012). In addition, the role of teachers is essential in environmental education. They are eligible and responsible for providing learners the skills, values, attitudes, knowledge and commitment for protecting and enhancing the environment, and to encourage students' active participation to resolve environmental issues (Khetani, Graham, Davies, Law, \& Simeonsson, 2015). Developing environmental orientations successfully requires environmentally educated teachers possessing competencies, pedagogy and scientific knowledge of adequate methods of teaching and approaches, environmental issues and their various causes, and scientific background. In contrast, lack of proficiency in environmental orientations, skills, and commitment of teachers will not lead to environmental change among students and schools (Rahi, 2015).

It is important to be aware and understand people's motivation to conserve the natural environment and preserve it. It is expected from the individuals being aware of their surroundings, to get worried about the effect of the environmental problems on themselves and give importance to the activities involved in their living environment. Moreover, individuals behave accordingly to get better results, which reflect the environmental orientations by their deeds. Approaching positively towards environment-related problems guides a consistent attitude towards the environment.

The educational environment is an important factor of students' behavior and achievements in curriculum activities. According to Fida, Ayuob, Alafari, Ismail, and Eldeek (2014), managing development changes is an essential part to improve environment worldviews. Throughout classroom learning, teachers should give a special concern to the classroom environment which can be divided into physical, intellectual and emotional contexts: (a) The physical context includes facilities, safety, and food and shelter, (b) The intellectual context involves learning with peers, daily practice, proper skills, and evidencebased knowledge, and (c) The emotional context provides students with security and reinforcement.

Kocak and Ewert (2017) argued that self-report surveys and scales are commonly used in studies regarding environmental orientations. The number of 
questionnaires or polls has been developed to measure abnormal environmental behavior, as well as students' attitudes over the past decades. To check the validity of these instruments to measure what they should really measure, attention has been given to their psychometric properties, since the examination of these properties has many advantages in including reliable, consistent and valid data collection.

When doing self-report surveys, respondents often tend to minimize their negative aspects and overstate positive aspects, which contribute a lot in examining and conducting analyses. Environmental Motives Scale had been used in past to identify the motivation beyond individuals' environmental interest and concern (Bruni, Chance, \& Schultz, 2012).

For instance, several instruments and measures may be developed to obtain students' participation in learning settings, and improve their skills of social, communal and environmental activities. Researchers in environmental educational frequently rely on questionnaires to provide data which can be useful as they offer a comparatively direct means of data collection. As a result of their study in developing a scale to measure values-based environmental concerns of students in early school years, Bruni, Chance, and Schultz (2012) asserted that understanding the differences in students' values, may lead educators and researchers and allow teachers to well understand the different facets of environmental orientations and concerns that students obtain and motivate them to take an action. In order to enable individuals to develop positive attitudes towards protection of environment, problems must be handled step-by-step. A number of theoretical plans have been developed to precise the gap between environmental orientations, in often pro-environmental behaviors.

Thereby, it is essential to measure the ecological information level available throughout a particular culture, and how individuals perceive ecological concerns that develop their environmental orientations. Hence, it is important to use an appropriate instrument for achieving such a purpose. Few researchers have been adopted the adaptation of different measuring instruments in a specific cultural context. It will not be sufficient to provide careful translation for validating a survey as linguistic terms are appropriate for the social and cultural conditions of the population selected. Therefore, there is a dire need to adopt psychometric properties and discuss it in a particular context as each society has its own attitudes, beliefs, and habits, reflecting the culture of a country. It is also important to carefully identify the individual values and norms to establish significant results based on each ecological concern. An essential element for the development of a valid and significantly useful tool is its length and the number of items it does contain. In addition, another important factor is the needed time to finish and answer all its questions. The use of a questionnaire for evaluating the environmental orientations of students in school-setting is an appropriate source when experiencing ecological concerns to investigate orientations affecting responsibilities toward ecology on the basis of its outcomes. Orientations and views are covert constructs and; therefore, they are not obvious responses and measured indirectly depending on the overt behavior, by the 
comprehensive direct-self report techniques, which is the most appropriate method using a questionnaire (Paulhus, \& Vazire 2007).

Ntanos, Kyriakopoulos, Skordoulis, Chalikias, and Arabatzis (2019) investigated citizens' perceptions towards the environment in Greece. This was based on the NEP Scale in order to recognize citizens' awareness by identifying the most significant items or aspects of the Scale. The researchers further explored if the NEP Scale scores are attributed to the participants' readiness to use renewable energy. The findings of the study revealed that the place where the participants lived in had a major impact on how they developed a new deep understanding of the environment. Also, the increase in the participants' scores was due to their readiness to get involved in renewable energy expansion activities and initiatives.

Bernstin and Szuster (2018) investigated to what extent a well-designed and appropriately used quantitative scale can help in empowering both individual and community. The researcher examined this hypothesis in light of many educators' perspectives that raised the idea of using a multi-item scale will not necessarily help children build up a deep understanding of environmentalism as a changing concept. To do so, the researchers began their study by previewing the different perspectives that tackled the NEP and other quantitative scales that examined the concept of environmentalism. Then, they examined how these different quantitative scales played a role in leading to a behavioral change. Toward the end of their study, the researchers suggested considering: technology, societal response, and nature as three factors that can help explore the reasons beyond students' different perspectives about environmentalism as a dynamic concept. The researchers recommended that educators and researchers need to consider such qualitative scales from different lenses rather than being judgmental.

Reyna, Bressán, Mola, and Belaus (2018) examined the psychometric properties of the New Ecological Paradigm (NEP) scale which is intended to measure students', orientations, perceptions and attitudes towards the environment. The researchers previewed previous studies to find out whether or not there were differences in the understanding of dimensionality although internal consistency was acceptable. The researchers used an exploratory factor analysis and an exploratory structural equation modelling as instruments of the study. The results showed that these techniques have assured that the bi-factor model which consisted of 11 items outperformed other models of psychometric properties were found to be effective. Also, the results revealed that a high level of environmental awareness was more strongly related to the participant' age than to gender.

Due to the widely spread and use of Dunlap and his colleagues' New Ecological Paradigm (NEP) scale, Amburgey and Thoman (2012) tested the advantages of this scale to determine if it should be dealt with as a single of 15-item scale (unidimensional), a group of five independent scales, or a set of five correlated subscales (multidimensional). The researchers used Confirmatory Factor 
Analysis (CFA) to examine these different assumptions. The findings of the study revealed that interrelated factors outperformed both single scale and independent factor structures. Accordingly, the researchers recommended that CFA should be used as a tool to better represent the structure of correlated scales.

Erkal, Kilic and Sahin (2012) highlighted the fact that educational activities can play an important role in helping educators and researchers to come up with appropriate solutions to environmental problems. They believed that it is important to enhance individuals' understanding of their rights and responsibilities toward the environment. Therefore, in their study, they aimed to explore how the university students think of the environment. The participants of the study were 213 students from two departments at Gazi University, the Business Administration Department and the Department of Family and Consumer Sciences Education. The researchers used the New Environmental Paradigm (NEP) scale to detect if the students in the Business Administration Department, who had taken no courses about the environment, had the same attitudes of those in the Department of Family and Consumer Sciences Education, who had taken an environment course. A 5-point Likert-type scale which consisted of 15 items was used. The results showed that students who had taken an environment course showed more positive attitudes and better understanding of the environment. Accordingly, the researches necessitated the need for enhancing individual awareness of environmental problems so that they work out solutions to these problems.

A measurable tool of 3 revisions of the original NEP scale was constructed by Dunlap, Van Liere, Mertig and Jones (2000) considering the environmental transition from local to global level of manipulation. The questions vary around three elements of environmental beliefs under the original NEP scale, which include (1) right of humans to rule over the rest of nature; (2) a belief in the ability of humans to upset the balance of nature; and (3) the presence of limits to growth. In more details, Dunlap and Van Liere (1978) have embarked on the development of NEP Scale, by observing the global changes that have led to the environmental problems and affecting the ecosystem. The 12-item NEP scale was developed to examine this environmental issue. Their effort was followed by a review and updating of the revised scale, consisting of 15- item, three of which were designed on each of its five facets (Dunlap et al. 2000). The revised NEP scale was then examined to ascertain if it was appropriate for use with 10 12 years old children (Manoli, Johnson, \& Dunlap, 2007).

This study aims to investigate the multidimensional nature of environmental orientations in lower elementary stage children, based on the New Ecological Paradigm (NEP) Scale. The ecological perceptions and the causes influencing the quality of the environment are assessed broadly by the NEP scale (Hosseinnezhad, 2017; Ntanos et al., 2019). A three-factor NEP appears to cover the most largely examined students and individuals' environmental orientations sufficiently, based on the aforementioned arguments. To this end, two primary objectives were proposed when measuring the psychometric properties; (1) to 
ascertain the baseline framework for environmental orientations in a schoolsetting, and (2) to indicate whether the 11-item NEP scale is appropriate for use in the school-setting in The United Nations Relief and Works Agency for Palestine Refugees (UNRWA) schools, Jordan. The UNRWA is one of the United Nations (UN) agencies established in 1949 to assist and protect the Palestinian refugees in health care, education and relief and social services (UNRWA, 2019).

\section{Material and Methods}

\subsection{Study Design}

The NEP Scale comprised 11 items for evaluating the three dimensions of environmental orientations. Following a descriptive correlational research design, the consented and anonymous questionnaire was administered to second and third grade students at UNRWA schools in Irbid City, Jordan. To this end, a total of 337 students agreed to participate in the survey. However, only 325 participants (113 females and 212 males) with ages of 7-9 years old, indicating very similar socio-demographic profiles were selected. They were eligible for the NEP measurement based on the complete questionnaire and appropriate responses, showing a $92.8 \%$ response rate. A total of 12 inconsistent and incomplete responses were identified, which were apparently not analysed and withdrawn from the study sample, as a part of the study. Furthermore, the data collection procedure was started during the first semester in the academic year 2018/2019.

\subsection{Data Validation}

The three dimensions: Eco-Crisis (EC), Rights of Nature (RN), and Human Exemptionalism (HE) of the 11-item questionnaire have been used for testing the validity and association of the constructs used in each dimension of the NEP in the Arabic context. A 3-point rating scale (disagree, neutral, and agree) was adopted to measure all responses. The questionnaire includes the relevant information regarding the questionnaire items and demographic information. The questionnaire was translated into Arabic ${ }^{1}$ because it is the communication language medium for teaching and learning at UNRWA schools in Jordan.

\subsection{Data Analysis}

AMOS, Analysis of covariance or causal modelling software within the statistical packages for social sciences version 23.0 has been utilized to analyse the collected data. An exploratory factor analysis and confirmatory factor analysis has been used to determine a common and latent construct, and to confirm the factor structure of the questionnaire, respectively.

\section{Results}

Using the extraction method of principal axis; the study has performed a principal component analysis with the association of oblique rotation after checking the internal consistency of the New Ecological Paradigm Scale. Table 1 demonstrates the reliability measures using Cronbach Alpha.

${ }^{1}$ The Arabic copy is to be requested from the author. 
Table 1: Cronbach Alpha

\begin{tabular}{lcc}
\hline Variable & scale item & Cronbach Alpha \\
\hline Eco-Crisis & $2,5,8,10$ & .81 \\
Rights of Nature & $1,4,7$ & .86 \\
Human Exemptionalism & $3,6,9,11$ & .84 \\
\hline
\end{tabular}

Table 2 shows the responses of students to the 11-item NEP scale. In general, most of them were agree with all scale statements. The findings have also shown that mostly students were in favor of that "If things don't change, we will have a big disaster in the environment soon" (52.6\%) followed by that "there are too many people on earth" (52\%), "people are supposed to rule over the rest of nature" $(51.4 \%)$, and (50.8\%) to the sixth item "nature is strong enough to handle the bad effects of our modern lifestyle".

Table 2: Responses Frequency Distribution of Children to the 11-Item NEP Scale

\begin{tabular}{|c|c|c|c|}
\hline \multirow{2}{*}{ scale item } & \multicolumn{3}{|c|}{ responses (\% of students) } \\
\hline & disagree & neutral & agree \\
\hline $\begin{array}{l}\text { 1.Plants and animals have as much right as people } \\
\text { to live. }\end{array}$ & $8.3 \%$ & $42.8 \%$ & $48.9 \%$ \\
\hline 2.There are too many people on earth. & $8.9 \%$ & $39.1 \%$ & $52 \%$ \\
\hline $\begin{array}{l}\text { 3.People are clever enough to keep from ruining the } \\
\text { earth. }\end{array}$ & $9.2 \%$ & $43.4 \%$ & $47.4 \%$ \\
\hline 4.People must still obey the laws of nature. & $12.9 \%$ & $41.5 \%$ & $45.5 \%$ \\
\hline 5.When people mess with nature it has bad results. & $7.4 \%$ & $42.8 \%$ & $49.8 \%$ \\
\hline $\begin{array}{l}\text { 6. Nature is strong enough to handle the good effects } \\
\text { of our modern lifestyle. }\end{array}$ & $7.1 \%$ & $42.2 \%$ & $50.8 \%$ \\
\hline $\begin{array}{l}\text { 7.People are supposed to rule over the rest of } \\
\text { nature. }\end{array}$ & $6.5 \%$ & $42.2 \%$ & $51.4 \%$ \\
\hline 8.People are treating nature badly. & $7.1 \%$ & $45.2 \%$ & $47.7 \%$ \\
\hline $\begin{array}{l}\text { 9.People will someday know enough about how } \\
\text { nature works to be able to control it. }\end{array}$ & $6.5 \%$ & $44.3 \%$ & $49.2 \%$ \\
\hline $\begin{array}{l}\text { 10.If things don't change, we will have a big disaster } \\
\text { in the environment soon. }\end{array}$ & $5.2 \%$ & $42.2 \%$ & $52.6 \%$ \\
\hline $\begin{array}{l}\text { 11. The so-called 'environmental crisis' facing people } \\
\text { has been blown out of proportion. }\end{array}$ & $8.1 \%$ & $43.1 \%$ & $48.8 \%$ \\
\hline
\end{tabular}

Factor loadings in exploratory factor analysis, and total variance each should be greater than .60 (Zikmund, Babin, Carr, \& Griffin, 2010). In Table 3, all scale items have met the criteria of .60 for loading. Hence, none of them were crossloaded. Also, Table 3 shows that the model total variance was $60.488 \%$. Therefore, one can claim that the results of exploratory factor analysis, were sufficient and accurate depending upon the above mentioned criteria. 
Table 3: Rotated Component Matrix

\begin{tabular}{lccc}
\hline \multirow{2}{*}{ item } & \multicolumn{3}{c}{ factor } \\
\cline { 2 - 4 } & $\mathbf{1 ( E C )}$ & $\mathbf{2 ( R N )}$ & $\mathbf{3 ( H E )}$ \\
\hline EC5 & .893 & & \\
EC3 & .885 & & \\
EC2 & .854 & & \\
EC10 & .846 & & \\
RN1 & & .863 & \\
RN7 & & .852 & \\
RN4 & & .849 & \\
HE8 & & & .879 \\
HE6 & & & .792 \\
HE9 & & & .777 \\
HE11 & & & .773 \\
Eigen Value & 3.761 & 3.069 & 2.244 \\
\% of Variance & 25.071 & 20.460 & 14.957 \\
Cumulative \% & 25.071 & 45.531 & 60.488 \\
\hline
\end{tabular}

Confirmatory factor analysis (CFA) Was performed to confirm the three factor model emerged from exploratory factor analysis (EFA). Results revealed that the three factor model was correctly adjusted to the data [i.e., $\chi^{2}=210.25$, $\mathrm{df}=51$; $\mathrm{p}<.01 \mathrm{x} 2 / \mathrm{df}=4.12$; comparative fit index $(\mathrm{CFI})=.92$ : root mean square error of approximation (RMSEA) $=.039]$. Table 4 shows the factor loadings validity means that all items of factors match the significant level $(\mathrm{p}<.0001)$. Table 4 also shows that construct items factor loading are matching the criteria of significant level. Therefore, the model factor loading value is greater than .60 .

Table 4: Construct Validity

\begin{tabular}{cccccc}
\hline item & path & Dimension & estimate & $\begin{array}{c}\text { standard } \\
\text { error }\end{array}$ & $\begin{array}{c}\boldsymbol{p} \\
\text { value }\end{array}$ \\
\hline RN1 & $<---$ & RN & .873 & .110 & .0001 \\
RN4 & $<---$ & RN & .871 & .013 & .0001 \\
RN7 & $<---$ & RN & .801 & .101 & .0001 \\
EC10 & $<---$ & EC & .772 & .131 & .0001 \\
EC2 & $<---$ & EC & .814 & .100 & .0001 \\
EC5 & $<---$ & EC & .742 & .132 & .0001 \\
EC3 & $<---$ & EC & .888 & .099 & .0001 \\
HE8 & $<---$ & HE & .815 & .133 & .0001 \\
HE6 & $<---$ & HE & .781 & .141 & .0001 \\
HE9 & $<---$ & HE & .770 & .135 & .0001 \\
HE11 & $<---$ & HE & .798 & .132 & .0001 \\
\hline
\end{tabular}

The regression coefficients have been presented in Table 5. The results have shown a significant and positive impact of rights of nature on NEP scales 
$(\beta=.318, p<.0001)$. Similarly, the results revealed a significant and positive impact of eco-crisis on NEP $(\beta=.456, p<.0001$. In addition, human exemptionalism is positively and significantly related with the NEP $(\beta=.247$, $\mathrm{p}<.002)$.

Table 5: Path Analysis

\begin{tabular}{lllcccc}
\hline & & & $\begin{array}{c}\text { estimate } \\
(\boldsymbol{\beta})\end{array}$ & $\begin{array}{c}\text { standard } \\
\text { error }\end{array}$ & $\begin{array}{c}\boldsymbol{t} \\
\text { stats }\end{array}$ & $\begin{array}{c}\boldsymbol{p} \\
\text { value }\end{array}$ \\
\hline $\mathrm{RN}$ & $<---$ & $\mathrm{NEP}$ & .318 & .079 & 4.008 & .0001 \\
$\mathrm{EC}$ & $<--$ & $\mathrm{NEP}$ & .456 & .117 & 3.886 & .0001 \\
$\mathrm{HE}$ & $<--$ & $\mathrm{NEP}$ & .247 & .124 & .382 & .002 \\
\hline
\end{tabular}

\section{Discussion}

The findings of the study reveal that item 6 denotes two different ideas in the ecological paradigm. One is the adverse influence while the other is the fact that nature is strong enough to mitigate and overcome these adverse impacts. However, in the context of UNRWA schools, it is found that the educational curriculum can be effective in advancing the individual knowledge pertaining to the effects caused by the practicing actions. This has been supported by the study of Spínola (2015), which stresses the development of educational curriculum for overcoming the shock and instilling effective response from the leaners. The study also found that the children competence and understanding of nature can be developed by the influence of the parents, media, as well as the peers.

Similar findings have been reported by Harraway, Broughton-Ansin, Deaker, Jowett, and Shephard (2012), which advocate it as an interesting area such as understating of nature will enable the child to understand that nature possesses the capability to absorb the produced garbage. The study also found that the development of promotion of the modern lifestyle also paves the path for the pollution and consumption of the globally produced items such as automobiles (Kopnina, 2011). The results of the focus group revealed concern as to whether this outcome of the modern lifestyle is observed globally or locally or it is confined to a certain locality or region only. It also revealed concerns related to the ability of nature such as whether its consumption tendency remains uniform or varies (Hosseinnezhad, 2017). The results of the present study show that the effect of the modern lifestyle and the response of nature might differ based on the schooling and understanding that is being developed with these environmental education programs. Moreover, parallel to the study of Izadpanahi and Tucker (2018), the new ecological paradigm is critiqued as an ineffective tool for resolving the arguments centered on the environmental ethics, especially the difference between the two approaches i.e. anthropocentric and eco-centric, and stating that the nature strength is not connected with the human's induced intrinsic value (Putu, 2017). Also, the idea related to the nature strength of weakness fails to focus on the effective state of emotional attachment of the child with nature (Ntanos et al., 2019). 
The results highlighted that there is an inadequate understanding among the children to some environmental issues such as the obedience of people the laws of nature and their bad treatment to it, which leads to their ambiguous interpretation to some environmental problems. The utilization of the quantitative tool is found to be limit the explicit examination of the NEP, which requires to be qualitatively analyzed for provisioning more factual, depth, and context specific. Therefore, future studies can expand the scope of the study by qualitatively analyzing the new ecological paradigm impact not only of children but all demographic groups in the country. Also, a similar notion may be investigated in other developing regions for devising stable and sturdy programs that provide value-added prospects. The researcher emphasized addressing the socio-context issues in the light of qualitative method using the variables such as environmental attitude, convictions as well as knowledge.

It is essential that the wider context of NEP-driven development and conceptualization are developed for treating the NEP as a scale to measure ecological views with respect to environmental orientations, attitudes, and behaviors. In addition, integration of the NEP scale in the Jordanian context can be regarded for predicting anticipating readiness to be considered in contingent valuation studies through advanced social-psychological patterns of environmental concerns, interest and orientations. Furthermore, this study has attempted to develop NEP scaling by resorting to format of 3- point Likert-type into children teaching with regard to making changes in content of wording to fit children's stage of development at UNRWA schools in Irbid city, Jordan. This NEP scaling approach for children learning is appreciated to be adapted for children in the second and third grades, which can encourage examinations of ecological worldviews or orientations. The actual and revised NEP scales are aspects of a specific organized space and time with respect to their formulation of issues and conceptual content in selected statements. Thereby, the use of unmodified scale in different cultures might create problems in the context of validity and reliability. The NEP statements can include contextual items on existing environmental concerns, which include global climate, water pollution, and sustainable development, reductions in biodiversity, deforestation, and air pollution. In addition, the concerns related to environment, which include conspicuous consumption, structural solutions, mechanical and technocratic solutions, and the mode and relations of mass production might be concerning among researchers.

The study results have added that though NEP is effective in measuring the efficacy of the environment in general, it still needs to be developed and adapted for infusing sensitivity in the socio-cultural context where education programs are being offered. In addition, it stresses the development of the NEP scale that is sensitive to the socio-cultural dynamics. This can be done through integration of the theories related to social context, political as well as institutional context. It also directs the future researches to explore the same phenomena using a qualitative method which instils children's beliefs, and the way these are formed in the socio-cultural context, which can help expand the subject knowledge horizon. 


\section{Conclusion}

The present study has evaluated the psychometric properties of the New Environmental Paradigm (NEP) Scale to ascertain its baseline framework for in a school-setting and indicate the appropriateness of 11-item test used in the Jordanian school setting. The main strengths of this study are the sample size which was big enough to conduct the statistical techniques and tests that have been utilized to analyze the collected data, and the number of items in the questionnaire that they do not take a long time to be answered by the students whom their concentration in a learning task such as reading a text, is usually short. In addition, the UNRWA was the scope of the school that gives special concerns to the environmental issues, as the UNRWA is one of the UN agencies who takes part in many environmental programs and campaigns worldwide. In spite of that, some limitations of the current study should be noted. First, it is limited to UNRWA second and third grade students in Irbid City in Jordan. Second, the scale items readability as the students are second and third graders and may suffer from reading or understanding difficulties. Third, the time of the data collection within the long study day may have an effect on students' seriousness to answer the questionnaire items; as the researcher doesn't work at their school.

The study results have provided a baseline for the sake of NEP scale. There is reduction in the potential response, when the responses are burdened. The response and completion rate are likely to be improved as a result of reduction in some items. In the current study, the findings revealed that the examination of the environmental education is limited to the given scale of NEP, which was consistently being applied for assessing its efficacy. Based on the results, it is found that the various socio-cultural dynamics may have impact on the utilization of the standardized quantitative measures particularly education related to the environment. Instead, the research has emphasized the development of a socio-cultural sensitive tool, which considers various social stimulators for assessing the popular environmental orientations. Therefore, the scale was recommended to be used as a valid instrument to assess students' environmental orientations in the lower elementary grades as well as evaluating the effectiveness of the environmental curriculum in developing such orientations. It is suggested that the NEP should be administered to another grades in the lower elementary stage in order to examine its psychometric properties and validity to be used in all grades in the lower elementary stage.

\section{Acknowledgements}

The author is grateful to those who contributed to developing the quality of this research. Further, no conflict of interest has been encountered during writing the research. 


\section{References}

Al Menhali, E. A., Khalid, K., \& Iyanna, S. (2018). Testing the psychometric properties of the environmental attitudes inventory on undergraduate students in the Arab context: A test-retest approach. PloS one, 13(5), e0195250. https://doi.org/10.1371/journal.pone. 0195250

Amburgey, J. W., \& Thoman, D. B. (2012). Dimensionality of the new ecological paradigm: Issues of factor structure and measurement. Environment and Behavior, 44(2), 235-256. https://doi.org/10.1177/0013916511402064

Barakat, R. S. (2015). The role of health and environment curriculum in developing environmental awareness of ninth grade basic students in governmental schools of Qalqilia Governorate according to teachers and students point of view. Unpublished MA Thesis, An-Najah National University, Nablus-Palestine. 161 p.

Bernstin, J., \& Szuster, B. (2019). The new environmental paradigm scale: reassessing the operationalization of contemporary environmentalism, The Journal of environmental Education, 50(2), 73-83, doi:10.1080/00958964. 2018.1512946

Bruni, C. M., Chance, R. C., \& Schultz, P. W. (2012). Measuring values-based environmental concerns in children: An environmental motives scale. The Journal of Environmental Education, 43(1), 1-15. https://doi.org/10.1080/00958964.2011. 583945

Danczyk, J., Jacobs, P., Montgomery, O., Jenkins, M., \& Farry, M. (2017). Testing the usability of a decision support system for increasing environmental awareness. In: 2017 IEEE Conference on cognitive and computational aspects of situation management (CogSIMA), (pp. 1-5). Savannah, GA.

Erkal, S., Kilic I., \& Sahin, H. (2012). Comparison of environmental attitudes of university students determined via the New Environmental Paradigm Scale according to the students' personal characteristics. Eurasian Journal of Educational Research, 49, 21-40.

Dean, A. J., Fielding, K. S.\& Newton, F. J. (2016). Community knowledge about water: who has better knowledge and is this associated with water-related behaviors and support for water-related policies?. PloS one, 11(7), e0159063. https://doi.org/10.1371/journal. pone. 0159063

Dunlap, R. E., Van Liere, K. D., Mertig, A. G., \& Jones, R. E. (2000). Measuring endorsement of the New Ecological Paradigm: A revised NEP scale. Journal of Social Issues, 56(3), 425-442. http:/ / doi: 10.1111/0022-4537.00176

Dunlap, R. E., \& Van Liere, K. D. (1978). The New Environmental Paradigm: A proposed measuring instrument and preliminary results. The Journal of Environmental Education, 9(4), 10-19. https:/ / doi.org/10.1080/00958964. 1978. 10801875

Halkos, G., Gkargkavouzi, A., \& Matsiori, S. (2018). Teachers' environmental knowledge and pro-environmental behavior: An application of CNS and EID scales. MPRA Paper 84505, University Library of Munich, Germany. Retrieved https://mpra.ub.uni-muenchen.de/84505/

Harraway, J., Broughton-Ansin, F., Deaker, L., Jowett, T., \& Shephard, K. (2012). Exploring the Use of the Revised New Ecological Paradigm Scale (NEP) to Monitor the Development of Students' Ecological Worldviews. The Journal of Environmental Education, 43(3), 177-191. http://doi:10.1080/00958964.2011.634450

Hosseinnezhad, F. (2017). A study of the new environmental paradigm scale in the context of Iran. European Journal of Sustainable Development Research, 1(2), article No. 14. http://doi.org/10.20897/ejosdr.201714 
Izadpanahi, P., \& Tucker, R. (2018). NEP (Children@School): An instrument for measuring environmental attitudes in middle childhood. Australian Journal of Environmental Education, 34(1), 61-79. http://doi:10.1017/aee.2017.25

Khetani, M. A., Graham, J. E., Davies, P. L., Law, M. C., \& Simeonsson, R. J. (2015). Psychometric properties of the young children's participation and environment measure. Archives of physical medicine and rehabilitation, 96(2), 307-316. https://doi.org/10.1016/j.apmr.2014.09.031

Kocak, F., \& Ewert, A. W. (2017). Psychometric properties of the Turkish version of the environmentally desirable responding scale (EDRS). Journal of Environmental Science and Management, 20(2), 68-76.

Kopnina, H. N. (2011). Applying the new ecological paradigm scale in the case of environmental education: Qualitative analysis of the ecological world view of Dutch children. Journal of Peace Education and Social Justice, 5, (3), 374-388.

Manoli, C. C., Johnson, M. B., \& Dunlap R. E .(2007). Assessing children's environmental worldviews: modifying and validating the New Ecological Paradigm Scale for use with children. The journal of Environmental Education, 38(4), 3-13. https://doi.org/10.3200/ JOEE.38.4.3-13

Miller, J., Brown, L., Hill, E., Shellman, A., Ramsing, R., \& Gómez, E. (2012). Measuring the educational impact of promoting environmental awareness in Kids (PEAK): The development and implementation of a new scale. In: Fisher, Cherie LeBlanc; Watts, Clifton E., Jr., eds. Proceedings of the 2010 Northeastern Recreation Research Symposium. Gen. Tech. Rep. NRS-P-94. Newtown Square, PA: US Department of Agriculture, Forest Service, Northern Research Station: 155-158.

Ntanos, S., Kyriakopoulos, G., Skordoulis, M., Chalikias, M., \& Arabatzis, G. (2019). An application of the New Environmental Paradigm (NEP) scale in a Greek context. Energies, 12(2), 239-? http:/ / doi:10.3390/en12020239

Paulhus, D. L., \& Vazire, S. (2007). The self-report method. In R. W. Robins, R.C. Fraley \& R.F. Krueger (Eds.), Handbook of research methods in personality psychology (pp. 224-239). London: The Guilford Press.

Putu, S. S. (2017). How Do Student Teachers' Beliefs Change When the New Ecological Paradigm Is Grounded into a Local Context Related to the Balinese Subak Landscape Heritage? International Journal of Environmental and Science Education, 12(3), 329-337. http://doi: 10.12973/ijese.2017.01230a

Rahi, A. S. (2015). Construction of an environmental awareness scale for prospective teachers. International Journal of Informative \& Futuristic Research, 2(11), 4353-4365. Retrieve from http://ijifr.com/pdfsave/21-08-2015950V2-E11-070.pdf

Reyna, C., Bressán, E., Mola, D., \& Belaus, A. (2018). Validating the structure of the New Ecological Paradigm Scale among Argentine citizens through different approaches. Pensamiento Psicológico, 16, (1), 107-118. http://doi:10.11144/Javeria nacali.PPSI16-1.vsne

Spínola, H. (2015). Environmental literacy comparison between students taught in Ecoschools and ordinary schools in the Madeira Island region of Portugal. Science education International, 26(3), 395-416. Retrieve from http://www.icaseonline.net/ sei/september2015/p8.pdf

UNRWA. (2019). Who we are. Retrieved from https:// www.unrwa.org/who-we-are

Zikmund, W. G., Babin, B.J. Carr, J.C. \& Griffin, M. (2010). Business research methods. CENGAGE Learning: USA. 